\title{
The Effect of Over-Voltage Protection by Shunt Capacitance and Its Optimization
}

\author{
Peng Cui \\ North China Electric Power University, Beijing, 102206 \\ alanncepu@foxmail.com
}

Keywords: Over-voltage protection; Resistance and capacitance absorption; Curve of power

\begin{abstract}
To investigate what an important role the shunt resistance branch circuit plays in overvoltage protection, this paper firstly analyzed the typical circuit with Kirchhoff's law. Then, by using Laplasse transform and inverse transform, the curves of voltage, current and power changing with time were obtained after the overvoltage occurs.After analyzing these curves, the essential role of RC protection playing was finally found, which can be seen as a temporary channel of circulation for overvoltage's prompt energy. In this channel, a large amount of energy will release in a short time, which played a very good effect on overvoltage protection.
\end{abstract}

\section{Introduction}

It is widely used in power system to realize over-voltage protection by shunt capacitor branch.However, take Fig.1 for example, according to the Kirchhoff voltage law, after shunt resistance branch, the over-voltage on ' $m n$ ', equals to ' $a b$ ' and ' $x y$ ', so , What is the protective effect of resistance capacitance branch exactly? Why is there still overvoltage but power systerm has been protected? In this paper, these issues will be explored.

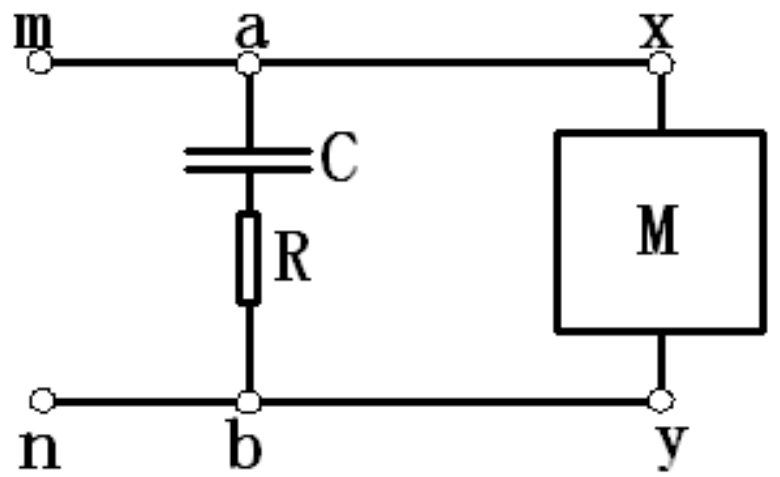

Figure 1. Parallel RC to protect Power network

\section{Causes of Over-voltage}

Switching Over-voltage. The transient over-voltage caused by the suddenly releasing of the energy of the inductive component when operating the AC side power supply.

In the transformer, there is a distributed capacitance between the two windings. If close the switch at the time when primary voltage came to the peak, there will be a high voltage coupled to the two winding and cause the instantaneous over-voltage via distributed capacitance. Besides, when the transformer is no-load, there is only the excitation current in the primary winding. And excitation current hysteresis supply voltage of about ninety degrees. When the supply voltage is zero, if suddenly break the switch, due to the sudden change of excitation current, the two winding will induce a high transient over-voltage, which can reach 6 times the peak value of the supply voltage, extremely harmful to power system. In addition to the Overvoltage on the transformer, when the converter is directly connected to the power supply, because of the sudden disconnection of adjacent load current, inductive electromotive force is generated on the inductance of the power 
supply circuit. The induced electromotive force and the voltage polarity of the power supply are exactly caused by the addition of the polarity.

Lightning (Surge) Over-voltage. Surge voltage, which is caused by lightning and other reasons, is called lightning surge overvoltage.

Turn off Over-voltage. Fully controlled devices operate at high frequencies. When the device is turned off, due to the rapid decrease of the forward current, the inductance of the circuit induces a voltage across the device.

Commutation Over-voltage. Because of the thyristor or the freewheeling diode which is connected in parallel with the full control device, the blocking ability can not be recovered immediately after the commutation phase, and therefore, a large reverse current flows through, so that the residual carrier is recovered. And when it restores the ability to block, reverse current decreases sharply. Such a sudden change in the current is due to the presence of line inductance, and will produce over-voltage in the thyristor cathode/anode, or anti parallel shunt diode on the full control of the device.

\section{Overvoltage Protection Measures}

AC side operation over-voltage is instantaneous peak over-voltage. The effective way to suppress the peak over-voltage is the shunt resistance capacitance absorption circuit. In Fig.1, in order to explore how to protect the network in the end is how to be protected, we then calculate the voltage, current and power of the $\mathrm{AB}$ branch, to get the specific effect of shunt $\mathrm{RC}$ on over-voltage protection.

\section{RC Protection Effect}

Modeling Idea. First, assign each parameter to calculate the voltage and current of the resistance capacitance absorbing branch.

Secondly, after the over-voltage occurred, make the curves of voltage and current changing over time. In the meanwhile, compare the voltage and current with the protected branch, to make a judgment that which is main influencing factors.

Finally calculate ]the power curve which can be applied to engineering practice。

Model Hypothesis. (1)Starting from the zero time;

(2)Power system can work normally;

(3)Operating overv-oltage occurs at 45 millisecond;

(4)Double exponential wave $u_{x}(t)=A U_{m}\left(1-e^{\left(-t / \tau_{1}\right)}\right) e^{\left(-t / \tau_{2}\right)} \varepsilon\left(t-t_{0}\right) \quad$.In this expression, $\tau_{1}=72.421 \mu \mathrm{s}, \tau_{2}=3282.12 \mu \mathrm{s}, A=1.037$, and to simplify the calculation, the $U_{m}$ is 5.

(5)The frequency of the system is $50 \mathrm{~Hz}$, and it is not affected by the operation over-voltage. In another word, system frequency remains unchanged;

(6)In the following calculations, the time is in seconds (s);

(7)Use modern excitation device, so over-voltage protection action time is $0.3 \mathrm{~s}$;

(8)The voltage, current and power in the following calculations are all the Per-unit value

Model Calculation. At the time of overvoltage generation, the network voltage is:

$$
u_{0}(t)=\sin \left(100 \pi \times 10^{-6} t\right)+A U_{m}\left(1-e^{-\left(t-t_{0}\right) / \tau_{1}}\right) e^{-\left(t-t_{0}\right) / \tau_{2}} \varepsilon\left(t-t_{0}\right)
$$

Take the voltage expression for Laplace transform, we can get: 
$U_{0}(s)=\frac{\omega}{s^{2}+\omega^{2}}+A U_{m}\left(\frac{1}{s+\frac{1}{\tau_{2}}}-\frac{1}{s+\frac{1}{\tau_{2}}+\frac{1}{\tau_{1}}}\right) e^{-s t_{0}}$

$U_{0}(s)=R C s \cdot U_{C}(s)-R C \cdot u\left(t_{0}^{-}\right)+U_{C}(s)$

From the previous hypothesis, $t_{0}=0.045 \mathrm{~s}$, Therefore we can calculate that $u\left(t_{0}^{-}\right)=1 \mathrm{~V}$. The current and voltage of the capacitor is:

$U_{C}(s)=\frac{1}{s C} I(s)+\frac{u\left(t_{0}^{-}\right)}{s}$

$I(s)=s C U_{C}(s)-C u\left(t_{0}^{-}\right)$

So we can get:

$U_{C}(s)=\frac{U_{0}(s)+R C}{1+R C s}$

$$
I(s)=\frac{s C U_{0}(s)+s R C^{2}}{1+R C s}-C
$$

Take $U_{0}(s)$ into it, after inverse Laplasse transform, and we can get voltage and current waveform.( Fig.2 \& Fig.3) :

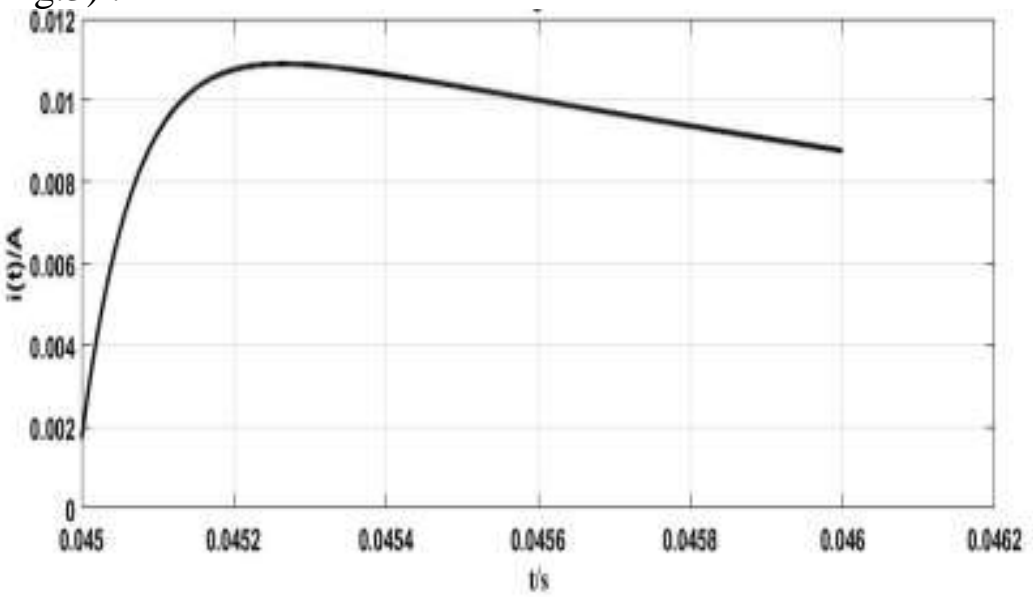

Figure 2.

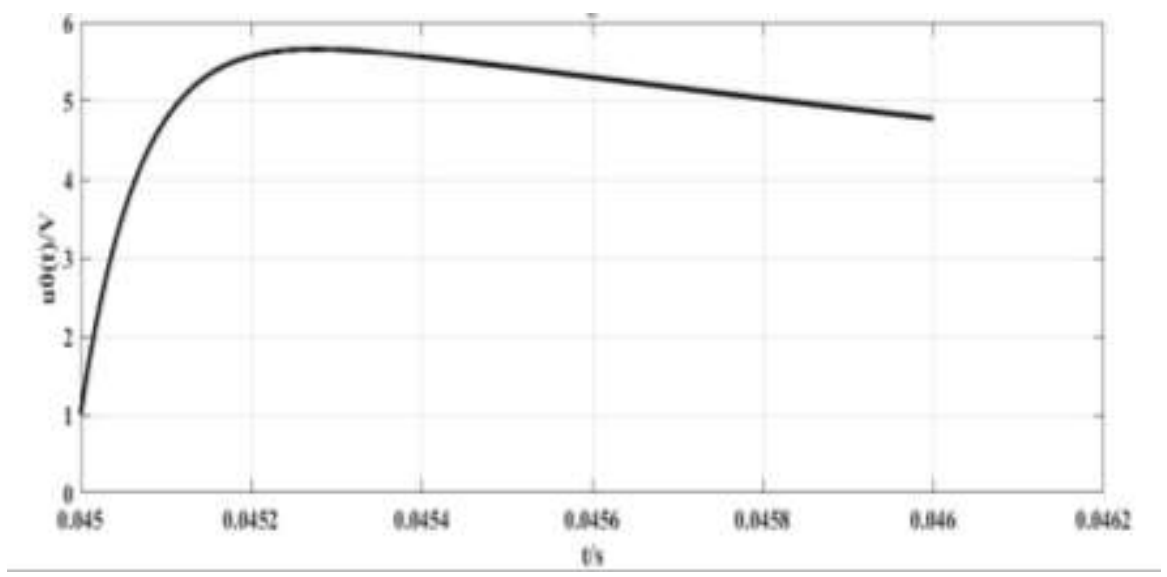

Figure 3. 


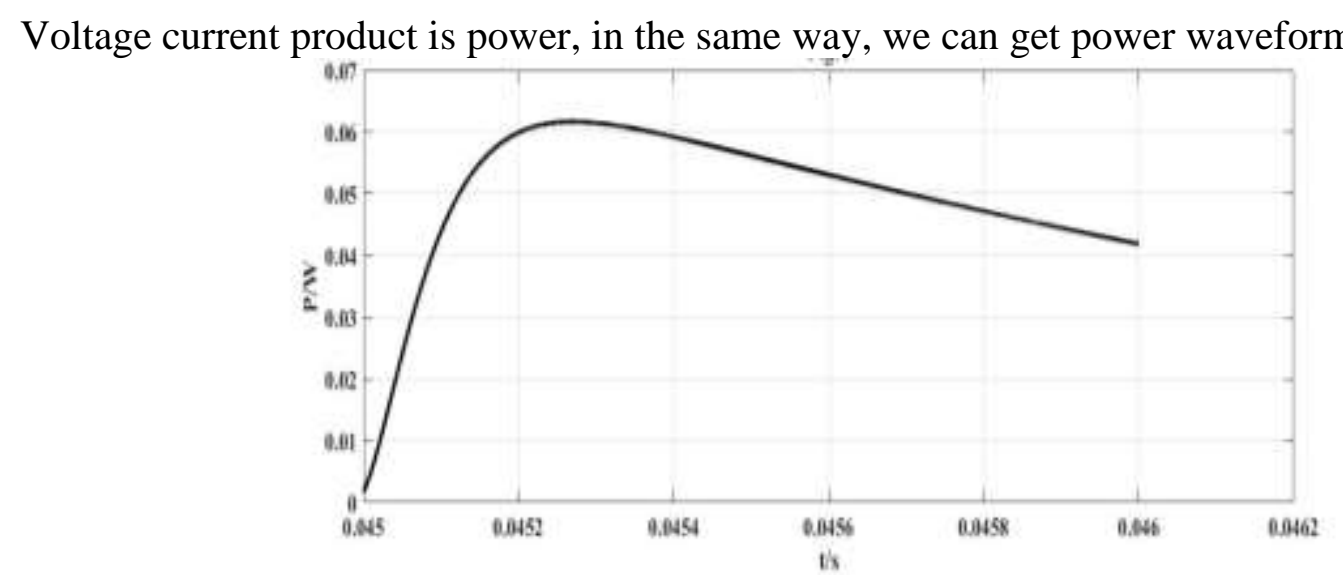

Figure 4.

\section{Conclusion}

Before over-voltage occurred, RC branch's power is 0.0017 , and the current flowing through the branch is 0.0017 . However, after over-voltage occurred, the current, voltage and power on the branch increase rapidly. In a very short time(270 microsecond), maximum current and power can be achieved 0.0109 and 0.0617 , which are 6.4and 36.3 times than the original. In the protected network, the inductance value can not be ignored, which means current mutation in a short time is impossible. So we can see, the role of the RC branch is to provide a channel for energy flow. When the voltage suddenly increases, the channel opens, and impact energy are released through this channel in a very short time, thus playing a protective role.

\section{References}

[1] Lee M H, Park C S. The Protection System Optimization of 154kV Shunt Capacitor Bank in Korea [J]. Journal of International Council on Electrical Engineering, 2013, 3(2):141-145.

[2] Chen J, Li H. Analysis of Switching Overvoltage in Regional Grid with Small Hydropower Plants [J]. Engineering, 2013, 05(9):19-23.

[3] Zhang X B, Wen D J, Sun Y M, et al. Lightning Overvoltage Analysis and Calculation of 330kV Overhead Transmission Line [J]. Applied Mechanics \& Materials, 2014, 556-562:1588-1591.

[4] Zha Fengwei; Song Zhiquan; Fu Peng;etal. Research on Reverse Recovery Transient of Parallel Thyristors for Fusion Power Supply[J]. Institute of Physics, 2014, 16(7):716.

[5] He Z Y, Deng Z F, Zha K P, et al. Study on overvoltage protection strategy of the thyristor valve overcurrent test equipment [J]. Proceedings of the Csee, 2006, 26(13):56-61.

[6] Yu Liu; Zhan Qingyu; Ying Huang; Experimental study on Lightning Induced Overvoltage of distribution lines [J]. Hans Publishers. 2013: 78-83.

[7] Liu Y, Duan C L. Study on Excitation Regulation System of Large Generator [J]. Power System Technology, 2006. 10 (30): 299-303.

[8] Nam S R, Kang S H, Park J K. Accurate phasor measurement for transmission line protection in the presence of shunt capacitor banks [J]. Electric Power Systems Research, 2007, 77(12):1646-1653.

[9] Patcharoen T, Ngaopitakkul A, Pothisarn C. Inrush current analysis for switching large shunt capacitor bank in a $230 \mathrm{kV}$ substation system[C]// International Conference on Electrical Machines and Systems. 2013:1325-1329.

[10]Nelson J H, Rostron J R, Luke D J. Two-stage, 161-kV, fuseless shunt capacitor bank[C]// T\&D Conference and Exposition, 2014 IEEE PES. IEEE, 2014:1-5.

[11]Nelson J H, Rostron J R, Luke D J. Two-stage, 161-kV, fuseless shunt capacitor bank[J]. 2014:1-5. 